\title{
Identification of topological magnetic order in a Weyl line ferromagnet
}

\author{
Guangming Cheng and Nan Yao
}

Princeton Institute for the Science and Technology of Materials, Princeton University, Princeton, New Jersey, United States

\section{Email: gcheng2@princeton.edu and nyao@princeton.edu}

Topological invariants are known to arise in the magnetic and electronic structures of crystals. [1-3] Despite the wealth of topological magnetic and electronic platforms, the discovery of a system which exhibits co-existing topology in both the magnetic order and the electronic band structure has remained elusive. Topological magnetic configurations such as magnetic vortices, skyrmions and magnetic bubbles have received enormous attention from the viewpoint of both fundamental science and promising applications in spintronic devices. The topological magnetic orders are considered to have strong antiinterference properties and are easily manipulated. Many techniques (referring to stray field, magnetic polarization, Lorentz force and weak lattice distortion) have been developed in characterizing the detailed magnetic configurations and the response of magnetic domains to the external magnetic fields, temperatures, etc. Among them, magnetic force microscopy (MFM)[4] and electron microscopes (EM), especially Lorentz transmission electron microscope (LTEM)[5], are effectively for visualizing magnetic domains from bulk to thin films. However, little effort has been made for the investigation of topological magnetic order in bulk samples because the domain structures are extremely sensitive to the sample geometry, induced stress and external magnetic field. In this study, we overcome the difficulty of sample preparation via focus ion beam (FIB) cutting. Here we report a real-space observation of a unique characteristic of topological magnetic order in a Weyl line ferromagnet as fabricated from a bulk single crystal.

To examine the surface properties of a L2 ${ }_{1}$ Co-based Heusler single crystal, we fabricated square-shaped lamellae with a fixed size of $15 \times 15 \times 1 \mathrm{um}^{3}$ from $<001>$ oriented bulk single crystal, shown in Fig. 1a. The edges (along the $\mathrm{x}$ and $\mathrm{y}$ axes) of the present sample correspond to the crystallographic axes of $<110>$ or $<100>$. Four separated electrodes were designed to be uniformly distributed on the corners of both the top and bottom surfaces, respectively. Figure $1 \mathrm{~b}$ shows the surface resistance as a function of temperature as measured by using the four surface electrodes placed along the top and bottom surfaces. To characterize the magnetic surface properties, we prepared square-shaped lamellae from $<001>$ oriented bulk single crystals so as to minimize the shape effect; a fixed side length (a) of $\sim 5$ um was utilized, while the thickness (t) was systematically varied. Figure 2 shows the surface domains from the top and bottom surfaces in the square-shaped lamellae with a sample thickness (t) of $1200 \mathrm{~nm}$, as measured by MFM. The samples were further thinned and examined via Fresnel imaging under LTEM and differential phase contrast imaging under LM-STEM.[6]

\section{References}

[1] I. Belopolski, K. Manna, D.S. Sanchez, G. Chang, B. Ernst, J. Yin, S.S. Zhang, T. Cochran, N. Shumiya, H. Zheng, Discovery of topological Weyl fermion lines and drumhead surface states in a room temperature magnet, Science 365(6459) (2019) 1278-1281.

[2] J.-X. Yin, W. Ma, T.A. Cochran, X. Xu, S.S. Zhang, H.-J. Tien, N. Shumiya, G. Cheng, K. Jiang, B. Lian, Z. Song, G. Chang, I. Belopolski, D. Multer, M. Litskevich, Z.-J. Cheng, X.P. Yang, B. Swidler, H. Zhou, H. Lin, T. Neupert, Z. Wang, N. Yao, T.-R. Chang, S. Jia, M. Zahid Hasan, Quantum-limit Chern topological magnetism in TbMn6Sn6, Nature 583(7817) (2020) 533-536. 
a
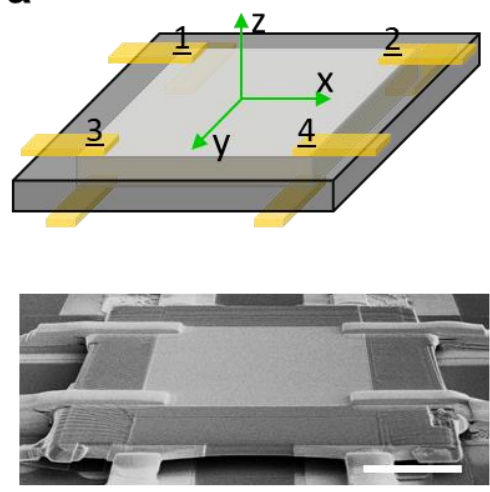

b

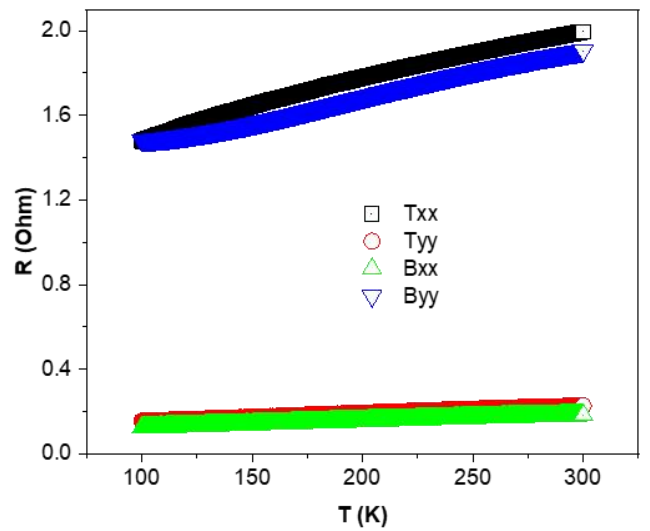

Figure 1. FIB cut lamellae for the physical measurement and microstructural characterization of a topological Weyl line ferromagnet. a, A square-shaped lamella fabricated by FIB cutting (top, a schematic; bottom, a SEM image). Scale bar, 5 um. b, Anisotropic changes of resistance measured from the top and bottom surfaces of a lamella with a thickness of $1 \mathrm{um}$.
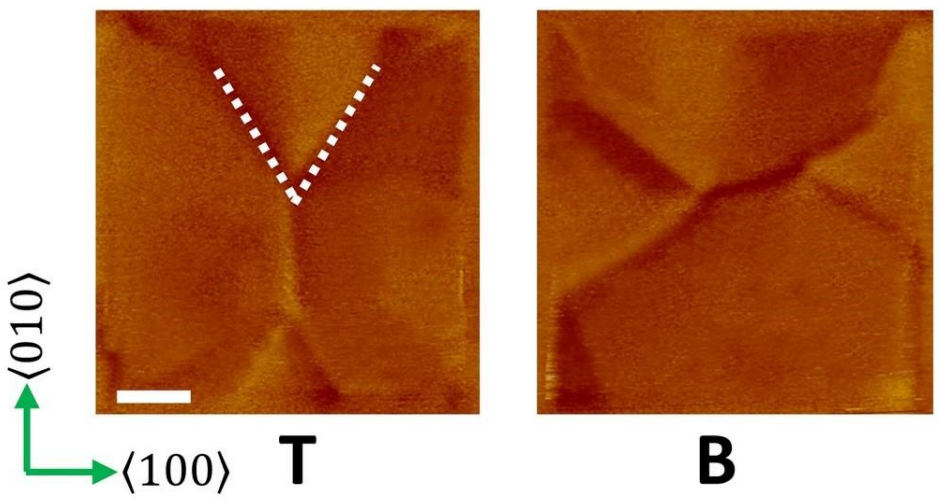

Figure 2. Experimental observation of the surface domains (top/T and bottom/B surfaces) in the lamella with a thickness of $1200 \mathrm{~nm}$, taken by MFM. Scale bar, $1 \mathrm{um}$.

[3] J.X. Yin, N. Shumiya, S. Mardanya, Q. Wang, S.S. Zhang, H.-J. Tien, D. Multer, Y. Jiang, G. Cheng, N. Yao, S. Wu, D. Wu, L. Deng, Z. Ye, R. He, G. Chang, Z. Liu, K. Jiang, Z. Wang, T. Neupert, A. Agarwal, T.-R. Chang, C.-W. Chu, H. Lei, M.Z. Hasan, Fermion-boson many-body interplay in a frustrated kagome paramagnet, Nature Communications 11(1) (2020) 4003.

[4] U. Hartmann, Magnetic force microscopy, Annual review of materials science 29(1) (1999) 53-87.

[5] A. Petford-Long, M. De Graef, Lorentz microscopy, Characterization of materials (2002) 1-15.

[6] The authors acknowledge the use of Princeton's Imaging and Analysis Center (IAC), which is partially supported by the Princeton Center for Complex Materials (PCCM), a National Science Foundation (NSF) Materials Research Science and Engineering Center (MRSEC; DMR-2011750). 